\title{
An Appraisal of Gracefulness Mediates the Negative Influence of Color Focality on Color Preference in Japan
}

\author{
Siyuan FANG, Yoshimasa TAWATSUJI and Tatsunori MATSUI
}

Waseda University, 1-6-1 Nishishinjuku, Shinjuku-ku, Tokyo 169-8050, Japan

\begin{abstract}
This study aimed to identify the psychological variables that can mediate an influence of color focality on color preference in modern Japanese culture and to specify the pattern of the mediated influence. The potential mediators were 22 appraisal dimensions and the psychological processing fluency (PPF) of colors. We conducted two experiments with native Japanese speakers, which measured these variables. Data analyses showed that gracefulness is the only appraisal dimension that can mediate an effect of color focality on color preference. Specifically, focality negatively influenced the appraisal of gracefulness and gracefulness positively impacted preference. Although a U-shaped quadratic relationship exists between focality and PPF, no relationship was found between PPF and preference. Therefore, PPF's role as a mediator is not supported. Our literature survey suggests that the focality-gracefulnesspreference relationship may be caused by the "noisy color phenomenon," which is a cultural phenomenon existing in many color-using areas in modern Japan.
\end{abstract}

Keywords: Color focality, Color preference, Gracefulness

\section{INTRODUCTION}

\subsection{Research Background and Objectives}

In most languages, the continuum of color perception is parceled up into a set of basic color categories (BCCs), such as red, green, yellow, blue, brown, pink, orange, grey, purple, black, and white in the English language [1,2], and akairo, midoriiro, kiiro, aoiro, chairo, pinkuiro, orenjiiro, haiiro, murasakiiro, kuroiro, and shiroiro in the Japanese language $[3,4]$. BCCs are natural categories that have prototype-centered structures. The colors that belong to a BCC differ in their focality. The focality of a color means the resemblance of the color to the prototype color of the category. In other words, the focality of a color signifies the typicality of the color as a member of the category, namely, the color's membership for the category $[5,6]$. Hence, color focality is also called "prototypicality" [7] or "typicality" [8] of colors in the literature.

In addition to focality, color has perceptual dimensions such as hue, lightness and saturation. However, in our daily lives, when talking about the color of an object, we often use a BCC to refer to the color, for example, "that red fruit", "that green tree", "that yellow bicycle", etc., rather than using the hue, lightness or saturation of the color. This indicates the practical importance of studying kansei/affective effects of color focality, which is a BCCdefined attribute, in the extensive area of kansei/affective engineering. To shed a new light on this research topic, the present study aims to identify the psychological variables that can mediate an influence of color focality on color preference in modern Japanese culture and to specify the pattern of that mediated influence. [Note 1]

Martindale and Moore's 1988 study [7] and Experiment 4 in Martindale, Moore, and Borkum's 1990 study [8] investigated the relationship between focality and preference of color stimuli for American English speakers. They used color chips selected from the Munsell Book of Color (Glossy Edition) as experimental stimuli. The focalities of the color chips were measured by Rosch [9] and Neumann and D'Agostino [10]. Their experimental results showed that their subjects tended to prefer colors of high focality to those of low focality. However, the two studies did not probe into the underlying mechanism, i.e., the mediating factors of the relationship between color focality and preference. To our knowledge, the present study is the first to explore the mediators of the focalitypreference relationship of colors in a specific culture.

Furthermore, the present study, which was conducted in the Japanese culture, is the only research ever conducted to study the relationship between color focality and preference in cultures other than the American culture. Thus, the present study is of great value to cross-cultural meta-analyses on the focality-preference relationship of colors. In addition, focality is, by definition, a continuous variable, but in Martindale and Moore's [7] and Martindale et al.'s [8] studies, color focality was defined as a discrete variable. This precluded their studies from reporting on the continuous pattern of the focality-preference relationship, namely how preference gradually changed along the continuum of 
focality. Our study resolved this problem by measuring color focality as a continuous variable using a modified version of Berlin and Kay's method [1].

In these ways, the present study provides new research perspectives and empirical findings that contribute to our understanding of how color focality influences humans' preference of colors, which is a kansei response vital to our daily uses of colors.

\subsection{Candidates for Mediating Variables}

Our study investigated two types of candidates for the variables, called "potential mediators", that possibly be capable of mediating an influence of color focality on color preference.

The first type consists of 22 appraisal dimensions (Table 1) of colors that Fang, Muramatsu, and Matsui [11] collected from the Japanese literature on color appraisals. According to the appraisal theories of emotions, humans constantly appraise objects they perceive based on a set of cognitive dimensions that are called "appraisal dimensions" in this study. Kuppens, Champagne, and Tuerlinckx [12] and Shuman, Sander, and Scherer [13] asserted that the appraisals on certain appraisal dimensions are able to influence the preference of the perceived object. [Note 2] The appraisal dimensions that can influence the preference for the stimuli used in our study, which are pure color chips, are pleasantness and beauty and congruence with moral standards proposed by Shuman et al. [13]. The former evaluates the hedonic feeling elicited by the perceived object, and the latter evaluates whether the object meets the moral norms or conventions defined in the culture.

The 22 appraisal dimensions used in the present study incorporated the abovementioned two dimensions. Specifically, beautiful-ugly and pleasant-unpleasant correspond to Shuman et al.'s [13] dimension of pleasantness and beauty, and positive-negative, successful-unsuccessful, graceful-awkward and true-false can be regarded as subdimensions of Shuman et al.'s [13] broad dimension of congruence with moral standards. The other appraisal dimensions that we use are descriptive assessments of the color qualia and their associations with other sensory modalities.

There are only a few studies on relationships between focality and appraisal dimensions. Babin and Babin's study [14] showed that the focality [Note 3] of a retail store affects consumers' appraisal of excitement and the excitement appraisal further impacts consumers' patronage intentions toward the store, i.e., their preference of the store. Also in research on consumer behavior, Goedertier, Dawar, Geuens, and Weijters's study [15] revealed that, in
Table 1: Original Japanese versions and English translations of the 22 color appraisal dimensions

\begin{tabular}{|c|c|}
\hline Original Japanese version & English translation \\
\hline 重い一軽い & heavy - light \\
\hline 明るいー暗い & light - dark \\
\hline 暖かい－涼しい & warm - cool \\
\hline 柔らかい－硬い & soft - hard \\
\hline うるさいー静かな & noisy - quiet \\
\hline 好きなー嫌いな & like - dislike \\
\hline 派手な一地味な & ornate - plain \\
\hline 強い-弱い & strong - weak \\
\hline 快いー不快な & pleasant - unpleasant \\
\hline きれいなー污い & clean - dirty \\
\hline 陽気な－陰気な & cheerful - gloomy \\
\hline 上品なー下品な & graceful - awkward \\
\hline 澄んだー濁った & clear - dull \\
\hline 静的な－動的な & static-dynamic \\
\hline 正しいー間違った & true - false \\
\hline 奇抜な－無難な & novel - ordinary \\
\hline 美しい-醜い & beautiful - ugly \\
\hline 安定したー不安定な & stable - changeable \\
\hline 成功したー失敗した & successful - unsuccessful \\
\hline 積極的な 一消極的な & positive - negative \\
\hline 緩んだー緊張した & relaxed - nervous \\
\hline 冷淡な－親切な & cruel - kind \\
\hline 受動的な - 能動的な & passive - active \\
\hline
\end{tabular}

some cases, the focality of a brand in its product category affects consumers' assessments of the brand-product fitness and risk of the newly developed products of the brand. These two assessments further influence consumers' preference of the products. Face evaluation literature has reported that the focality of a person's face affects the appraisal of the person's trustworthiness [16], and the trustworthiness appraisal closely relates with the preference of the face [17]. In view of these reports, although we found no literature on relationships between focality and appraisal dimensions of colors, we deem it interesting to explore whether such relationships exist in terms of colors.

The second type of potential mediators was the "psychological processing fluency (PPF)" of colors. To explain Martindale and Moore's [7] experimental results, Reber, Schwarz, and Winkielman [18] argued that high-focality colors were more likely to be preferred than low-focality colors because higher focality of a color could make the mental processing of the color more fluent. However, this fluency hypothesis faces challenges as stimuli that are easy to process could also be felt to be boring and thus less attractive than more complex stimuli [19]. In the present study, we experimentally tested this debated role of PPF as a mediator between focality and preference of colors. Because PPF is an abstract concept in Reber et al.'s [18] hypothesis, we need to determine an operative indicator of 
PPF to measure it quantitatively. We chose short-term memory (STM) accuracy of our color stimuli as the operative indicator of their PPF because the STM of a stimulus reflects multiple properties of the stimulus that we consider closely relate with the fluency of the mental processing of the stimulus, e.g., linguistic codability [20-22], perceptual discriminability [20], and ecological relevance [23].

The present study conducted two psychological experiments. Experiment 1 measured the PPF and the focality of the color stimuli and Experiment 2 measured the preference and the 22 color appraisal dimensions of the stimuli. The details of Experiment 1 have been published in Fang and Matsui's 2018 paper [24], so the present paper only briefly describes the parts of the procedure and results of this experiment that directly relate to the main objectives of this study. Using the data obtained in the two experiments, we tested whether the two types of potential mediators could actually mediate an effect of color focality on color preference.

\section{EXPERIMENT 1}

\subsection{Participants, Stimuli, and Environment}

Twenty-two native Japanese speakers (11 males and 11 females of ages $M=31.45$ and $S D=14.34$ ) who were either undergraduate or graduate students at Waseda University participated in this experiment. None of them reported having any color-related art experience. They all passed the Ishihara Color Vision Test (38 plates, International Edition) and no one reported having color-vision deficiencies. We obtained informed consent of participation from all participants. All experimental procedures in this study comply with the ethical standards of the Office of Research Ethics of Waseda University.

A color array designed by Rosch [25] was used in this experiment. Figure 1 shows its layout which was formed by chips in the Munsell Book of Color (Glossy Edition). The array was made of cardboard $(58.5 \mathrm{~cm} \times 28.5 \mathrm{~cm})$ and had color chips embedded in the white surface. Thirty colorswhich are the colors within the grey area in Figure 1 and are called "test colors" for short-were tested in the formal trials of Session 1. These colors were mainly selected as test colors for the following reasons: 1) these colors covered a large contiguous region on the array, so they likely sampled the entire continuum of color focality, and 2) our knowledge of the Japanese language suggested that these colors could sample a relatively large number of Japanese BCCs. Each test color was mounted on the white surface of a $5.0 \mathrm{~cm} \times 5.0 \mathrm{~cm}$ piece of cardboard when presented to the participants.

The experiment was performed indoors with fluorescent lighting (National FHF 32EXN-H, daylight color, color temperature: $5000 \mathrm{~K}$ ). The illuminance was 744 lux measured by a HIOKI FT3424 lux meter (JIS C 16091 General Class AA, DIN 5032-7 Class B). This lighting condition accorded with the convention of this research field in which STM of colors was tested in natural daylight or fluorescent light that simulated daylight. The experimenter and the participant being tested sat opposite each other at the experiment table. Every test color was presented at a distance of $50 \mathrm{~cm}$ from the participant's eyes. A cardboard wall was erected along the middle of the table in order that the participant was unable to see the experimenter's face and behaviors.

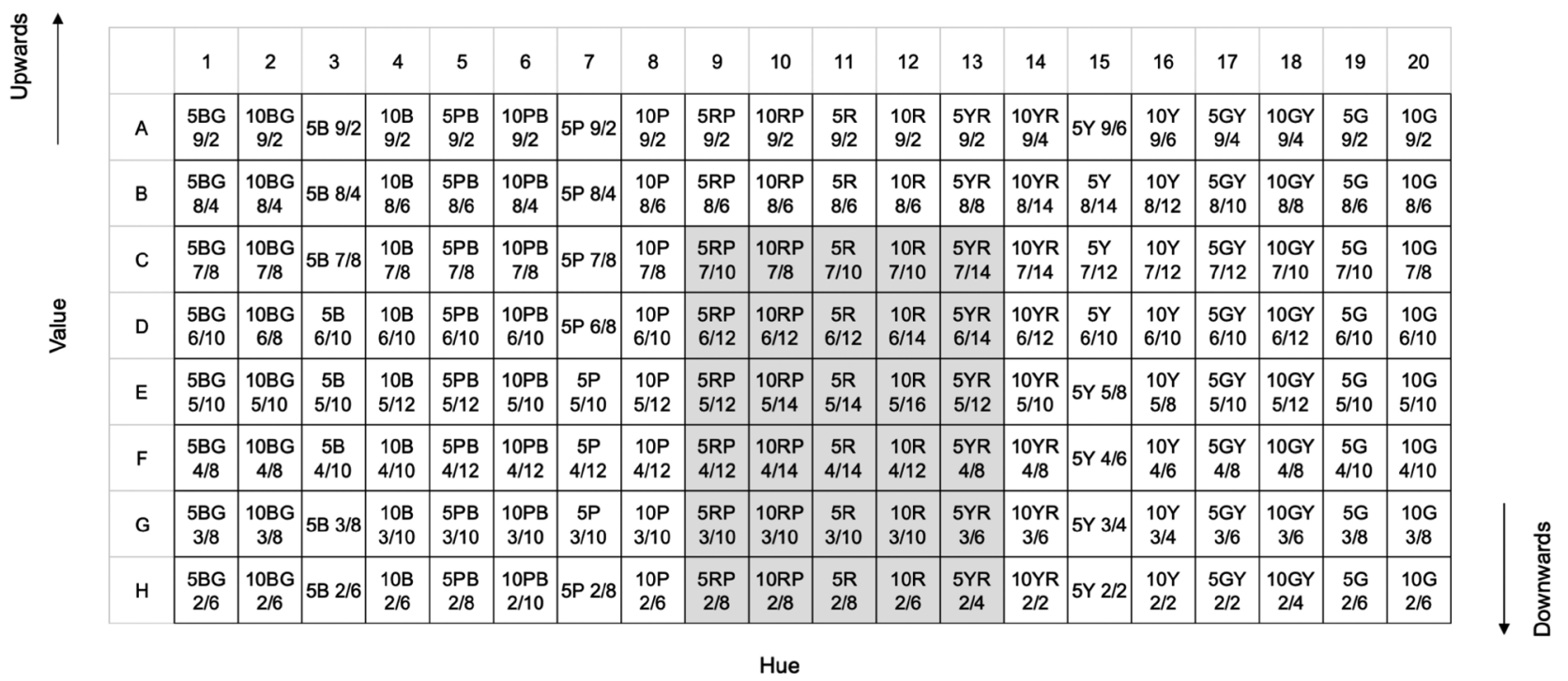

Figure 1: Layout of the color array used in Experiment 1

Test colors are those within the grey area. 


\subsection{Procedure}

Experiment 1 consisted of two sessions. Session 1 quantified the PPF of the test colors by measuring the participants' STM accuracy of the test colors. Session 2 measured the focality of the test colors.

Session 1 used a procedure similar to that developed by Rosch [25]. It consisted of 33 trials. In each trial, a test color was presented to the participant for $5 \mathrm{~s}$ and was then retrieved. After $30 \mathrm{~s}$, the color array was presented to the participant and the participant was asked to report which color in the array he/she thought was the one that had been previously presented. Prior to the formal experiment, there were two training trials which used two other test colors. Following the training trials, each test color was tested at least once and in random order for each participant. Thus, for each participant, there were three repeated trials, which were intended to prevent the participant from using a strategy of excluding the already tested colors in the color-searching task.

Session 2 elicited the coverages of the six BCCs, namely akairo [red], pinkuiro [pink], kiiro [yellow], orenjiiro [orange], chairo [brown], and murasakiiro [purple]. The status of these categories as BCCs in the Japanese language was empirically substantiated by Uchikawa and Boynton [3] and Kuriki et al. [4]. In this session, the participant being tested was required to report all colors that he/she thought could be named by each of the six BCCs (Task 1), and then the colors that he/she thought were the best examples of the six BCCs (Task 2). Multiple answers were allowed for each BCC, but the participant was instructed to narrow down his/her choices as much as possible. This method of quantifying focality of colors is a revised version of Berlin and Kay's [1] method.

\subsection{Computation of Variables}

Memory Accuracy Score. Using the data obtained in Session 1, we specified the memory accuracy score (MAS) of each test color as the percentage of the trials in which the participants correctly recognized the test color in the color array.

Focality Score. We used the data obtained from Session 2 to determine the categorical membership and the focality of each test color. We first computed the Red Index, the Pink Index, the Yellow Index, the Orange Index, the Brown Index, and the Purple Index of each test color. The Red Index of a color was computed by dividing the number of participants who named the color as akairo $[$ red] by the total number of the participants. The other five indexes were similarly defined. Next, we defined the Overall Index (OI) of the color as the greatest of the six indexes of the color. We classified a color into the BCC akairo $[$ red] if its OI was its Red Index, into the BCC pinkuiro [pink] if its OI was its Pink Index, and so forth. Figure 2 shows the coverages of the six BCCs and the OIs of the color stimuli. Since the six single-term-derived indexes measured the inter-participant consistency of color naming regarding each color term and the OI is the largest among them, we defined the focality score (FS) of the color as the value of its OI. Then, we examined the psychological appropriateness of this definition using the participants' responses in Task 2 , that is, the task of selecting best examples of the BCCs, in Session 2. The logic is that if OI of a color really represents its focality, namely, the typicality of the color as a member of its BCC, the colors of high OIs (designated as OIs $\geq .80$ ) should be selected as the best examples of their BCCs by the majority of the participants. The selection data show that this is the case: Regarding the BCCs akairo, kiiro and

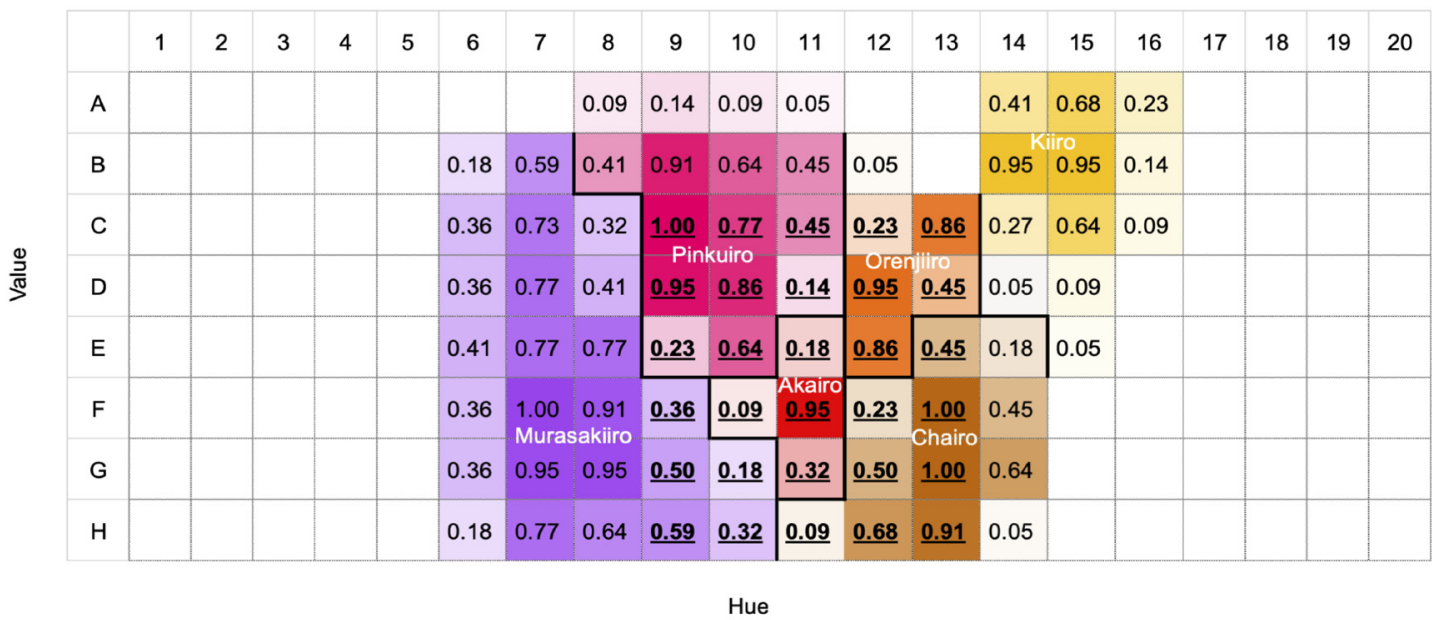

Figure 2: Partition of the six BCCs and the OIs of the color stimuli

Color depth signifies the magnitude of the OI.

The OIs of the test colors are bolded and underlined.

For the chip E15, Brown Index $=$ Orange Index $=.05$. 
orenjiiro, the high-OI colors were selected as the best examples by $100 \%$ of the participants. Regarding pinkurio, this percentage was $83 \%$; chairo $81 \%$, and murasakiiro $75 \%$. This means that most participants considered the high-OI colors the best examples of the BCCs, which substantiates the psychological appropriateness of our definition of FS.

\section{EXPERIMENT 2}

\subsection{Participants, Stimuli, and Environment}

Thirty-two native Japanese speakers who were either undergraduate or graduate students at Waseda University participated in the experiment. They all passed the Ishihara Color Vision Test (38 plates, International Edition) and none reported having color-vision deficiencies. We obtained informed consent for participation from all participants. The data obtained from three participants were excluded from the data analyses because they reported having had a colorrelated art experience. The data obtained from the remaining 29 participants (14 males and 15 females of ages $M=28.41$ and $S D=12.24$ ) were used in the data analyses.

The experiment used the 30 test colors that were used in Experiment 1 as stimuli. The sizes of the effects of color focality on color preference that Martindale and Moore [7] and Martindale et al. [8] detected, which were measured by the variance of color preference explained by color focality in their ANOVAs, have a mean of .244. To detect an effect of this size with $\alpha=.05$ and $1-\beta$ (i.e., statistical power $)=.80$ in a two-tail case, our continuous-variable experiment paradigm needs at least 29 samples, i.e., test colors (calculated using $G^{*}$ Power, version 3.1). This means that our study has a reasonable sample size. The experimental environment was also the same as in Experiment 1.

\subsection{Procedure}

The experiment consisted of 30 trials. In each trial, a test color was presented to the participant, who was asked to rate it using 22 seven-point Likert scales, which represented the 22 color appraisal dimensions (e.g., for the appraisal dimension heavy-light, extremely heavy $=3$, very heavy $=2$, a bit heavy $=1$, neither heavy nor light $=0$, a bit light $=-1$, very light $=-2$, and extremely light $=-3$ ), and another seven-point Likert scale like-dislike, which measured the degree of preference. We used Fukuda and Fukuda's [26] Japanese expressions for the seven markers on each scale. Although no time limitation was imposed on the participant's ratings, he/she was asked to rate the test colors without deliberation. The order of the rating scales was randomly determined for each participant.
To remove the afterimages, we presented a grey chip (N 5.5/) for $30 \mathrm{~s}$ between every two trials and asked the participants to watch it. We used the N 5.5/ chip because it had the middlemost value (i.e., degree of lightness) among the achromatic chips in the Munsell Book of Colors (Glossy Edition). Before the formal experiment began, a training trial was conducted in which the $\mathrm{N} \mathrm{5.5/}$ chip was used as the test color. Following the training trial, each test color was tested once and in random order for each participant. The participants did their ratings using the online survey tool SurveyMonkey. The interface was set to be achromatic.

\subsection{Computation of Variables}

Preference Score. We defined the preference score (PS) of each test color as the average of the rating scores across the participants on the scale like-dislike. We chose to use this Likert scale-based definition because it was used by most previous studies that measured color preference, including Martindale and Moore's [7] and Martindale et al.'s [8] studies.

Color Appraisal Scores. We defined each color appraisal score (CAS) of a test color as the average of the rating scores across the participants on the corresponding color appraisal dimension.

\section{STATISTICAL ANALYSES AND RESULTS}

\subsection{Testing Potential Mediating Effect of Color Appraisal Dimensions}

For each color appraisal dimension, we tested whether it can actually mediate color focality and color preference by examining its relationship with color focality and that with color preference.

We looked into the relationship between color focality and each color appraisal dimension by performing Pearson correlation tests between FS and each CAS of the test colors at Bonferroni adjusted $\alpha=.05 / 22=.0023$. We found a significant negative linear relationship between FS and the gracefulness score (GS), $r(28)=-.58, p<.001$, as plotted in Figure 3(A). The GS of a color was computed as the average rating score of the color on the appraisal dimension graceful-awkward. No other CAS showed any statistically significant relationship with FS.

Next, we investigated the relationship between each appraisal dimension and color preference by performing Pearson correlation tests between each CAS and PS of the test colors at Bonferroni adjusted $\alpha=.0023$. A significant positive linear relationship was found between GS and PS, $r(28)=.62, p<.001$, as plotted in Figure 3(B). Because 
(A)

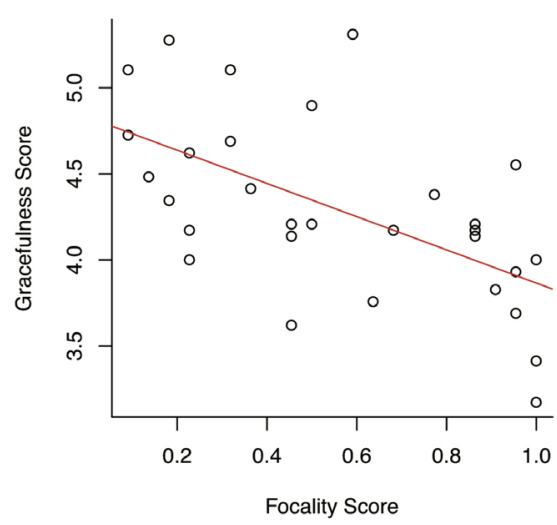

(C)

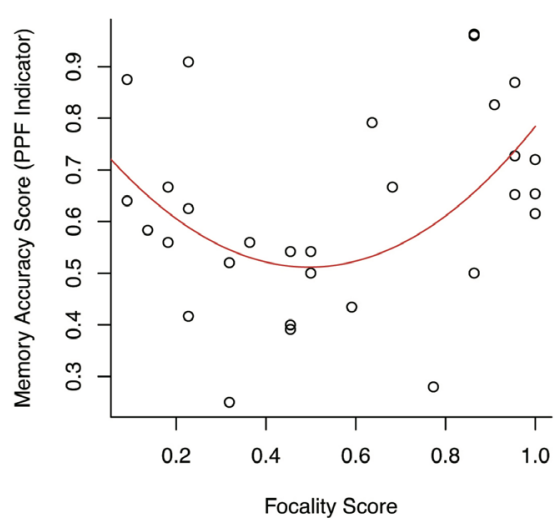

(B)

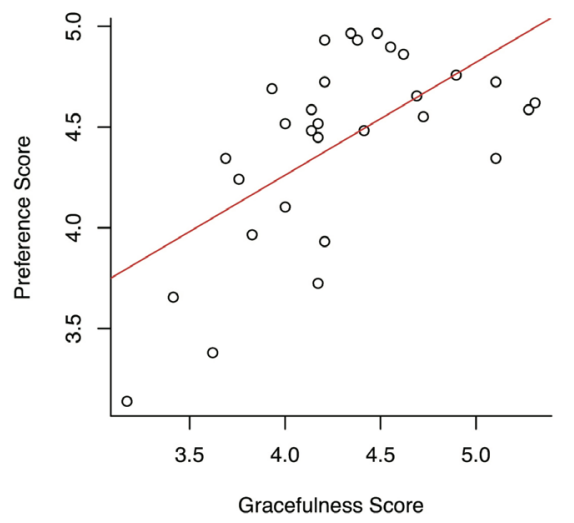

(D)

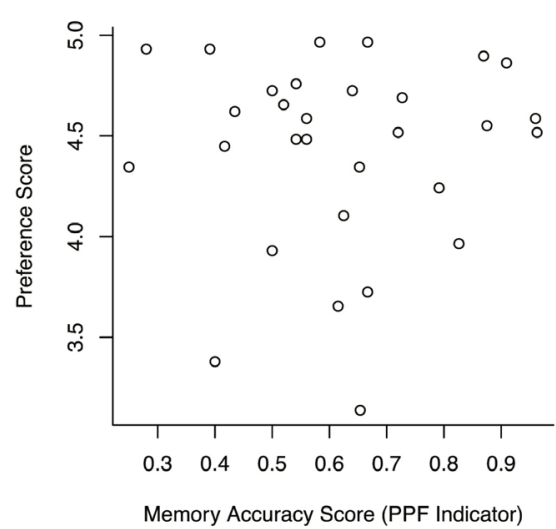

Figure 3: Plots of the data and the statistically significant regression models in terms of (A) focality score and gracefulness score, (B) gracefulness score and preference score, (C) focality score and memory accuracy score (i.e., indicator of PPF), and (D) memory accuracy score and preference score.

GS has a significant relationship with both FS and PS, it is reasonable to contend that GS bridges FS and PS. With regard to other CASs, some have a significant correlation with PS. These correlations are listed in Table 2. However, none of them have a significant relationship with FS. Thus, these CASs cannot mediate FS and PS.

Table 2: Color appraisal scores that have a significant correlation with preference score

\begin{tabular}{lccr}
\hline Color appraisal score & Color appraisal dimension & $r$ & $p$ \\
\hline lightness score & light-dark & .555 & .001 \\
ornateness score & ornate-plain & .668 & $<.001$ \\
pleasantness score & pleasant-unpleasant & .910 & $<.001$ \\
cleanness score & clean-dirty & .889 & $<.001$ \\
cheerfulness score & cheerful-gloomy & .629 & $<.001$ \\
clearness score & clear-dull & .673 & $<.001$ \\
trueness score & true-false & .793 & $<.001$ \\
novelty score & novel-ordinary & .581 & $<.001$ \\
beauty score & beautiful-ugly & .922 & $<.001$ \\
successfulness score & successful-unsuccessful & .818 & $<.001$ \\
positivity score & positive-negative & .657 & $<.001$ \\
activity score & passive-active & .549 & .002 \\
\hline
\end{tabular}

\subsection{Testing Potential Mediating Effect of Color PPF}

We tested the hypothesized mediating effect of color PPF by examining its relationship with color focality and that with color preference. For all tests, $\alpha=.05$.

We first investigated the relationship between color focality and PPF. The Pearson correlation test showed no significant linear relationship between FS and MAS, which is the operational indicator of PPF, $r(28)=.26, p=.171$. However, we discerned a U-shaped relationship between FS and MAS in the scatter plot, i.e., Figure 3(C), and a quadratic regression analysis revealed that this relationship is significant, $F(2,27)=4.19, R^{2}=.24, p=.026$ (the linear term: $\beta=.23, t(27)=-2.11, p=.045$; the quadratic term: $\beta=.58, t(27)=2.46, p=.021)$. The Cohen's [27] $f^{2}$ of the regression model is 0.31 , which means a relatively large effect according to Cohen's [27] criteria. This model indicates that MAS is highest at the two ends of the continuum of FS and decreases as FS moves towards the medium region from either end.

Next, we looked into the relationship between MAS and PS by examining the scatter plot of the MAS and PS data, 
i.e., Figure 3(D), and performing a Pearson correlation test and a quadratic regression analysis. We discerned no trend in the plot and the analyses showed no significant linear or quadratic relationship between MAS and PS (the correlation test: $r(28)=.04, p=.845$; the quadratic regression analysis: $\left.F(2,27)=.59, R^{2}=.04, p=.562\right)$. These results indicate that PPF is unable to bridge FS and PS, which counters Reber et al.'s [18] fluency hypothesis.

\subsection{Mediation Analysis for Indirect Effect of Color Focality on Color Preference}

The statistical analyses described above show that FS negatively correlates with GS and that GS positively correlates with PS. This indicates that there exists an indirect effect of FS on PS which is mediated by GS. We then evaluated this putative indirect effect by following Zhao, Lynch Jr., and Chen's [28] revised procedure of Baron and Kenny's [29] mediation analysis. The model of the indirect effect consisted of the Equations 1 and 2, in which $i_{1}$ and $i_{2}$ are the intercepts, $a, b$ and $c$ the unstandardized regression coefficients, and $e_{1}$ and $e_{2}$ the random errors.

$$
\begin{aligned}
& G S=i_{1}+a^{*} F S+e_{1} \\
& P S=i_{2}+b^{*} G S+c^{*} F S+e_{2}
\end{aligned}
$$

We performed a bias corrected and accelerated (BCa) bootstrapping $[30,31]$ to test the statistical significance of the overall mediation effect of GS and estimate the effect size, which is $a^{*} b$, using the $\mathrm{R}$ package $M B E S S$ (version 4.6.0). The result, computed on 1000 bootstrapped samples, is that $a^{*} b=-.59, \mathrm{BCa} 95 \%$ CI $[-1.29,-.23]$. This indicates a significant negative indirect effect of FS on PS transmitted by GS because zero fall outside the CI. Next, we performed a regression analysis for Equation 1 and for Equation 2 respectively. The results of the two regression analyses show that FS negatively predicts GS, and GS positively predicts PS, and FS cannot predict PS when the impact of the mediator GS on PS has been partialled out, $a=-.96, p=.001, b=.61, p=.001$, $c=.14, p=.603$. Figure 4 shows the resulted mediation model. We also ran a Pearson correlation test directly on

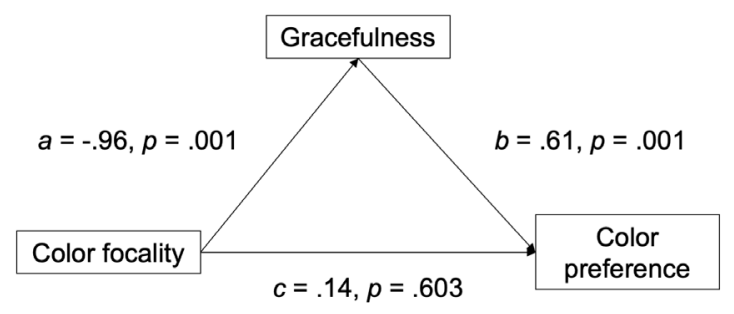

Figure 4: The resulted mediation model the FS data and PS data. The result showed an overall negative trend, but it did not achieve statistical significance, $r(28)=-.30, p=.108$. This is probably because the FS-PS regression model contained both the noises in the FS-GS data and those in the GS-PS data. According to Zhao et al.'s [28] typology of mediations, the mediation by GS of the FS-to-PS effect is an indirect-only mediation.

\section{DISCUSSION}

\subsection{Focality-Gracefulness-Preference Relationship}

The experimental results show that, with regard to the color appraisal dimensions, there is a negative linear relationship between FS and GS and a positive linear relationship between GS and PS. No CAS other than GS was found to have a significant relationship with FS, although some of them had a significant relationship with PS. This means that within the color appraisal dimensions, only gracefulness can mediate color focality and color preference. Regarding PPF, the experimental results show that although MAS as the operational indicator of PPF has a significant U-shaped quadratic relationship with FS, no significant relationship exists between MAS and PS. This means that PPF's role as a mediator between color focality and color preference is not supported in this study. All in all, our experiments show that the gracefulness is the only psychological variable that can mediate between color focality and color preference in modern Japanese culture. This finding can be interpreted using the psychological model of visual aesthetic preference proposed by Leder, Belke, Oeberst, and Augustin [32].

In the model, the psychological mechanism of visual aesthetic evaluation mainly consists of three modules: the module of visual information processing, the module of memory, and the module of affective evaluation. The module of visual information processing has four sequential stages. The first stage, called "Perceptual Analyses," processes simple perceptual features of visual stimuli, for example, contrast, complexity and color. The second stage, called "Implicit Memory Integration," evaluates properties of visual stimuli that can be extracted unconsciously from the module of memory, such as familiarity, prototypicality, and the peak-shift effects of objects. The third and fourth stages relate with art appreciation and are thus irrelevant to the present study. The module of affective evaluation evaluates the preference of the stimuli at any stage during the visual information processing. 
Using this model to construe our findings, because the formation of BCCs is largely constrained by the perceptual attributes of colors [33,34], color focality can be regarded as being processed at the Perceptual Analyses Stage. Regarding the appraisal dimension gracefulness, because the appraisal of gracefulness depends on the specific culture and can be performed without conscious effort, it likely occurs at the Implicit Memory Integration Stage, that is, one stage after the processing of color focality. Then, according to Leder et al.'s [32] description, the affective evaluation module takes the outcomes of the visual information processing as inputs, and outputs the preference evaluation. Thus, the FS-GS and GS-PS relationships detected in this study can be interpreted using Leder et al.'s [32] model as this influence chain: color focality negatively influences the appraisal of gracefulness and the gracefulness appraisal positively influences color preference. Our mediation analysis also supported this indirect effect.

\subsection{Noisy Color Phenomenon: A Possible Cause of the Focality-Gracefulness-Preference Relationship}

Because the gracefulness appraisal is a culture-dependent appraisal, we performed a literature survey to search for cues of the cultural derivation of the focality-gracefulnesspreference relationship detected in this study. The literature survey covered not only academic books and papers but also online fashion shopping sites, fashion news sites, and fashion blogs. The in-text citations of these online or printed sources, except those of Mitsuboshi's and Yoshimura's papers [35-37], start with an S, and their reference list is provided as a supplementary file [38].

From the literature survey, we noticed that a "noisy color phenomenon (NCP)" exists in many color-related areas such as city planning, apparel design, and cosmetics in present-day Japan. We speculate that the NCP is a cause of the focality-gracefulness-preference relationship.

The term NCP originated in the 1960s in the realm of city planning and referred to the phenomenon that highfocality colors, especially those in the range of warm colors, tended to be sensed as being "noisy," that is, flashy and gaudy, and was thus felt to be unpleasant and dissonant with surrounding colors [35-37].

According to Mitsuboshi's studies [35,36], the NCP received public attention after the occurrence of the "three major incidents of NCP" in the 1980s. The most famous was the Setagaya McDonald's Incident which happened in 1985 in the Setagaya District in Tokyo. In this incident, McDonald's erected a huge neon sign on the roof of a tall building with its brand colors-red, white, and yellow-flashing repeatedly. Protests organized by nearby residents broke out, which claimed that the neon sign had brought unbearable color pollution. In the other two incidents, Tokyo's city buses and a BigCamera shopping mall were painted very salient and hot colors which made local residents feel unpleasant. In view of these incidents, Mitsuboshi suggested refraining from using high-focality warm colors in public transport carriers, buildings, and advertising boards. Some enterprises have adopted this strategy. For example, McDonald's and 7-Eleven replaced the original high-focality red color with darkish brown on the logo plates in a portion of their branch stores. Also, the vending machines in some districts were placed in wooden cases that covered their pure red exterior $[35,36]$.

In addition, regarding clothing, Japanese people are accustomed to dressing in low-focality colors in their daily lives [S1]. High-focality colors tend to be regarded as gaudy, distasteful, and, therefore, unsuitable for many occasions such as dating [S2], working in offices [S3-S5], or attending children's school entrance ceremonies as their guardians [S6,S7]. High-focality colors are also avoided in facial make-up [S8,S9] and nail polish [S10].

These reports demonstrated the wide-spread existence of the NCP in color-using situations in Japan. According to Yoshimura's [37] following argument, the NCP reflects the Japanese tradition in which modesty is highly valued:

There was a tradition in Japan that "bitter colors" are considered beautiful. "Bitter colors" refer to subtle and composite colors that are composed of various hues such as red, yellow, green, blue, and purple that contain a bit of grey... In the Japanese language, there are proverbs that tell people that keeping a low profile is a graceful manner of behavior. These include proverbs such as "unexpected piles [or nails] are to be struck" and "hawks that have brains hide nails" as well as proverbs derived from law provisions legislated in the feudal periods, such as "there is no winner in a quarrel." In the traditional Japanese culture in which modesty is a highly respected virtue, "bitter colors" contain a sense of beauty as modest composite colors. (pp.19-20; translated from Japanese into English by the authors of the present paper)

Based on the findings of our literature survey described above, we speculate that our Japanese subjects unconsciously acquired the disgraceful impression of high-focality colors detected in our experiments from their everyday color-using experiences in which the NCP had long become a cultural routine. 
If this is true, then the focality-gracefulness-preference relationships detected in cultures without the NCP, especially cultures that favor high-focality colors, will have patterns different from that detected in Japan. Martindale and Moore's [7] and Martindale et al.'s [8] experimental results support this inference, which demonstrated that American people preferred high-focality colors to low-focality colors, as described in Section 1.1. Their experimental results probably reflect the 1980s fashion trend to dress in bright and bold colors, i.e., high-focality colors, in the English-speaking countries [S11-S14]. We also found reports showing that the cultures in Scandinavian countries [S15], Korea [S1,S16-S20] and London [S21] favor at least some high-focality colors and thus presumably have no NCP. Experimental evidence for these and other non-NCP cultures is needed to clarify the diversity of the focality-gracefulnesspreference relationship of colors across cultures.

\section{SUMMARY AND FUTURE WORK}

This study aimed to identify the psychological variables that could mediate an influence of color focality on color preference in modern Japanese culture and to specify the pattern of the mediated influence. Twenty-two color appraisal dimensions and color PPF were investigated as candidates for the mediating variables.

We conducted two psychological experiments with native Japanese speakers. Experiment 1 measured the focality and PPF of the color stimuli. Experiment 2 quantified the appraisal dimensions and preference of the stimuli. Data analyses showed that the color appraisal dimension gracefulness mediates a negative relationship between color focality and color preference. More precisely, using Leder et al.'s [32] model as theoretical framework, color focality negatively influences the gracefulness appraisal and the gracefulness appraisal positively impacts color preference. Regarding other color appraisal dimensions, although some of them have a significant relationship with color preference, none of them significantly relates with color focality. This means that gracefulness is the only appraisal dimension that can mediate color focality and color preference. With regard to PPF, although there is a U-shaped quadratic relationship between color focality and PPF, no significant relationship exists between PPF and color preference. Therefore, PPF can hardly be a mediator between color focality and color preference at least for the Japanese people. Our literature survey suggests that this focality-gracefulness-preference relationship may be caused by the NCP, which exists extensively in the color-using fields in the Japanese society.
In terms of practical applications in kansei/affective engineering, the results of this study imply the need to take into account the possibility that high-focality colors may elicit negative feelings when we conceive designs that assume Japanese users, especially in the realms of architecture, city planning, and fashion.

Several concerns should be taken into consideration in interpreting our experimental results. First, our results did not rule out the existence of feedbacks from preference and gracefulness appraisal of colors to color focality. However, even if such top-down effects exist, they are expected to be weaker than the bottom-up processing which is essential for perception and survival [39]. More neuroscience evidence is needed to obtain a detailed temporal architecture of the focality-gracefulnesspreference relationship. Also, there were perhaps mediators between color focality and preference that lay beyond our literature survey. We will continue searching for such mediators. In addition, like other psychological studies on color focality, the present study used just several dozens of Munsell glossy color chips as test colors. It would be important to replicate the present study using other test colors and color coordinate systems.

The results of this study also implicate the following topics for future explorations. As explained in Section 5, the variance of the pattern of the focality-gracefulnesspreference relationship across cultures can be investigated by conducting experiments in other cultures using our experimental paradigm. From the perspective of cognitive psychology, it would also be important to investigate the mediating variables between focality and preference in natural category domains other than color, e.g., shapes, phonemes, textures and facial expressions.

\section{ACKNOWLEDGEMENTS}

This work was supported by Waseda University Grant for Special Research Projects (Project number: 2017S-207).

\section{NOTES}

1. Parts of the experimental results and discussions of this study were presented at the 2018 Congress of the International Association of Empirical Aesthetics (Toronto, Canada, August 2018) and the 2018 Interim Meeting of the International Colour Association (AIC) (Lisbon, Portugal, September 2018) with an AIC proceedings paper [40].

2. Preference is termed "valence" in Kuppens et al.'s study [12] and "macro-valence" in Shuman et al.'s study [13].

3. Focality is termed "typicality" or "prototypicality" in the studies [14-17]. 


\section{REFERENCES}

1. Berlin, B., and Kay, P.; Basic color terms: Their universality and evolution, University of California Press, Oakland, California, pp.1-10, 1991 (first published in 1969 by University of California Press).

2. Sturges, J., and Whitfield, T.W.A.; Locating basic colours in the munsell space, Color Research \& Application, 20(6), pp.364-376, 1995.

3. Uchikawa, K., and Boynton, R. M.; Categorical color perception of Japanese observers: Comparison with that of Americans, Vision Research, 27(10), pp.18251833, 1987.

4. Kuriki, I., Lange, R., Muto, Y., Brown, A. M., Fukuda, K., Tokunaga, R., Lindsey, D.T., Uchikawa, K., and Shioiri, S.; The modern Japanese color lexicon, Journal of Vision, 17(3), pp.1-18, 2017.

5. Rosch, E. H.; Natural categories, Cognitive Psychology, 4(3), pp.328-350, 1973.

6. Kay, P., and McDaniel, C. K.; The linguistic significance of the meanings of basic color terms, Language, 54(3), pp.610-646, 1978.

7. Martindale, C., and Moore, K.; Priming, prototypicality, and preference. Journal of Experimental Psychology: Human Perception and Performance, 14(4), pp.661-670, 1988.

8. Martindale, C., Moore, K., and Borkum, J.; Aesthetic preference: Anomalous findings for Berlyne's psychobiological theory, The American Journal of Psychology, 103(1), pp.53-80, 1990.

9. Rosch, E.H.; The nature of mental codes for color categories, Journal of Experimental Psychology: Human Perception and Performance, 1(4), pp.303322, 1975.

10. Neumann, K.M., and D'Agostino, P.R.; Specificity of mental color codes, The American Journal of Psychology, 94(3), pp.451-459, 1981.

11. Fang, S., Muramatsu, K., and Matsui, T.; Experimental study of aesthetic evaluation to multi-color stimuli using semantic differential method, Transactions of Japan Society of Kansei Engineering, 14(1), pp.37-47, 2015.

12. Kuppens, P., Champagne, D., and Tuerlinck, F.; The dynamic interplay between appraisal and core affect in daily life, Frontiers in Psychology, 3, 380, 2012.

13. Shuman, V., Sander, D., and Scherer, K. R.; Levels of valence, Frontiers in Psychology, 4, 261, 2013.

14. Babin, B. J., and Babin, L.; Seeking something different? A model of schema typicality, consumer affect, purchase intentions and perceived shopping value, Journal of Business Research, 54(2), pp.89-96, 2001.
15. Goedertiera, F., Dawarb, N., Geuensc, M., and Weijters, B.; Brand typicality and distant novel extension acceptance: How risk-reduction counters low category fit, Journal of Business Research, 68(1), pp.157-165, 2015.

16. Todorov, A., Olivola, C.Y., Dotsch, R., and Mende-Siedlecki, P.; Social attributions from faces: Determinants, consequences, accuracy, and functional significance, Annual Review of Psychology, 66, pp.519-545, 2015.

17. Oosterhof, N.N., and Todorov, A.; The functional basis of face evaluation, Proceedings of the National Academy of Sciences, 105(32), pp.11087-11092, 2008.

18. Reber, R., Schwarz, N., and Winkielman, P.; Processing fluency and aesthetic pleasure: Is Beauty in the perceiver's processing experience? Personality and Social Psychology Review, 8(4), pp.364-382, 2004.

19. Reber, R.; Processing fluency, aesthetic pleasure, and culturally shared Taste. In: Shimamura, A.P., and Palmer, S.E. (ed.), Aesthetic science: Connecting mind, brain, and experience, Oxford University Press, New York, pp.223-249, 2012.

20. Brown, R.W., and Lenneberg, E.H.; A study in language and cognition, The Journal of Abnormal and Social Psychology, 49(3), pp.454-462, 1954.

21. Lucy, J.A., and Shweder, R.A.; Whorf and his critics: Linguistic and nonlinguistic influences on color memory, American Anthropologist, 81(3), pp.581-615, 1979.

22. Lucy, J.A., and Shweder, R.A.; The effect of incidental conversation on memory for focal colors, American Anthropologist, 90(4), pp.923-931, 1988.

23. McCarthy, C.R. J.; Ecologically relevant stimuli and color memory, The Journal of General Psychology, 117(4), pp.369-377, 1990.

24. Fang, S., and Matsui, T.; An experimental study on the continuous patterns of the influence of color focality on short-term memory performance of colors, International Journal of Affective Engineering, 17(3), pp.193-204, 2018.

25. Rosch, E. H.; Universals in color naming and memory, Journal of Experimental Psychology, 93(1), pp.10-20, 1972.

26. Fukuda, T., and Fukuda, R.; Ningen Kogaku Gaido: Kansei o Kagakusuru Hoho (Zohoban) [Introductory ergonomics: A scientific approach to kansei (Extended edition)], Saiensu-Sha, Tokyo, pp.132-133, 2009. (in Japanese)

27. Cohen, J.; Statistical Power analysis for the behavioral sciences (2nd edition), Lawrence Erlbaum Associates, 
Hillsdale, New Jersey, pp.410-414, 1988.

28. Zhao, X., Lynch, J.G., and Chen, Q. Jr.; Reconsidering baron and kenny: Myths and truths about mediation analysis, Journal of Consumer Research, 37(2), pp.197-206, 2010.

29. Baronm R.M., and Kenny, D.A.; The moderatormediator variable distinction in social psychological research: Conceptual, strategic, and statistical considerations, Journal of Personality and Social Psychology, 51(6), pp.1173-1182, 1986.

30. Efron, B.; Better bootstrap confidence intervals, Journal of the American Statistical Association, 82(397), pp.171-185, 1987.

31. Efron, B., and Tibshirani, R. J.; An introduction to the bootstrap, CRC Press, Boca Raton, Florida, pp.184188, 1993.

32. Leder, H., Belke, B., Oeberst, A., and Augustin, D.; A model of aesthetic appreciation and aesthetic judgments, British Journal of Psychology, 95(4), pp.489-508, 2004.

33. Jameson, K., and D'Andrade, R.G.; It's not really red, green, yellow, blue: An inquiry into perceptual color space. In: Hardin, C.L., and Maffi, L. (eds.), Color categories in thought and language, Cambridge University Press, Cambridge, pp.295-319, 1997.

34. Regier, T., Kay, P., and Khetarpal, N.; Color naming reflects optimal partitions of color space, Proceedings of the National Academy of Sciences, 104(4), pp.1436-1441, 2007.

35. Mitsuboshi, M.; A line of noisy color pollutions in Japan and their solutions, Bulletin of the Institute for Humanities Research of Kanagawa University, 46, pp.35-51, 2011. (in Japanese)

36. Mitsuboshi, M.; Public color pollutions and issues of landscape: Reality and solutions, Bulletin of the Institute for Humanities Research of Kanagawa University, 50, pp.41-67, 2013. (in Japanese)

37. Yoshimura, K.; Cultural differences between English and Japanese synesthetic expressions with color terms: The real meaning of synesthesia and Japanese situation-centered expressions for onomatopoeia, Journal of Inquiry and Research of Kansai Gaidai College, 86, pp.19-37, 2007. (in Japanese)
38. Fang, S.; Supplementary Reference List.docx, Figshare, Online Resource (posted on 12/6/2020), https://doi. org/10.6084/m9.figshare.13337849.v1.

39. Rolls, E. T.; A biased activation theory of the cognitive and attentional modulation of emotion, Frontiers in Human Neuroscience, 7, 74, 2013.

40. Fang, S., and Matsui, T.; Experimental investigation into the mediating variables of the relationship between colour focality and colour preference. In: International Colour Association (ed.) Proceedings of the International Colour Association Conference 2018, pp.743-748, 2018.

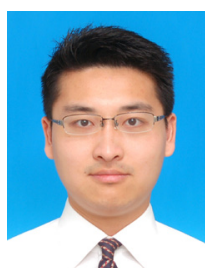

Siyuan FANG (Member)

Siyuan Fang is an assistant professor in data science at Global Education Center, Waseda University, Tokyo, Japan. He received Doctor of Human Sciences from Graduate School of Human Sciences, Waseda University in 2018. His doctoral supervisor is Prof. Tatsunori Matsui. His research interests are psychological mechanisms of kansei/affective evaluations of visual information, interactions among categorization, memory and aesthetic attitudes, and computational aesthetics.

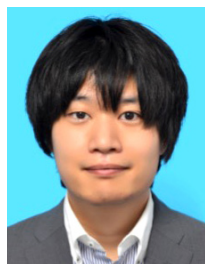

Yoshimasa TAWATSUJI (Member)

Yoshimasa Tawatsuji is an Assistant Professor at Global Education Center, Waseda University. He received his Ph.D. in Human Sciences from Waseda University in 2020. He was previously a Research Associate at the School of Human Sciences, Waseda University. His research interests include human-agent interaction, model of others, neural mechanism of uncanny valley, and emotions.

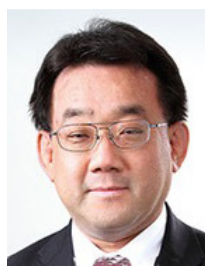

Tatsunori MATSUI (Member)

Tatsunori Matsui graduated from Department of Mathematics, School of Science and Engineering, Waseda University in 1988, and received Dr., Sci. in 1994, and has worked as a research assistant at Waseda University, an assistant professor at Tokyo Gakugei University, an associate professor at the University of Electro-Communications, an associate professor at Faculty of Human Sciences, Waseda University, and a professor at Faculty of Human Sciences, Waseda University since 2007. His research interests include Kansei Information Science, Knowledge Information Science, Skill Science, and Learning Support Systems. 